\title{
Situação atual e o potencial para o desenvolvimento do cultivo de polvo no Brasil
}

\author{
Actual situation and potential for development of octopus culture in Brazil
}

\author{
Marco Antonio Igarashi
}

Engenheiro de pesca, PhD, professor do Departamento de Engenharia de Pesca da Universidade Federal do Ceará (UFC), Londrina, PR - Brasil, e-mail: igarashi@ufc.br

\section{Resumo}

O potencial do cultivo do polvo é analisado nesta revisão levando em consideração as características biológicas, a tecnologia de cultivo, assim como os aspectos econômicos, os quais são relevantes para o futuro desenvolvimento do seu cultivo comercial, que envolve a captura de juvenis e o cultivo em confinamento no Brasil. A fácil adaptação a condições de cativeiro, a alta taxa de crescimento, a aceitação de alimentos naturais de baixo valor e o alto preço no mercado são fatores considerados para o cultivo comercial. Resultados das pesquisas obtidos em projetos piloto são também citados, em linhas gerais, para reforçar a possibilidade de cultivo do polvo.

Palavras-chave: Cultivo. Polvo. Engorda.

\begin{abstract}
The potential of the octopus breeding is evaluated in this review, taking into consideration biological characteristics and the technology for breed, as well as some economic aspects, which may be relevant for the future development of octopus farming in Brazil. The commercial breeding of octopus involves capturing juveniles and growing them in confinement. This possibility has been considered in determined regions of Brazil, where young can be captured for growout. The easy adaptation to captivity conditions, acceptance of low value natural foods, and high market price are the requirements to be considered as a candidate for commercial breeding. The results obtained from pilot project are also cited on general headlines, needed to improve the possibility of octopus breeding.
\end{abstract}

Keywords: Breeding. Octopus. Growth.

Rev. Acad., Ciênc. Agrár. Ambient., Curitiba, v. 8, n. 4, p. 417-427, out./dez. 2010 


\section{Introdução}

A classe Cephalopoda compreende cerca de 700 espécies (SWEENEY; ROPER, 1998). O gênero Octopus inclui aproximadamente 200 espécies, e o Octopus vulgaris Cuvier, 1797 (ordem Octopoda, subordem Incirrata) é uma das mais importantes espécies em termos de desembarque e valor comercial (VAZ-PIRES et al., 2004).

O polvo comum (O. vulgaris) é uma espécie que tem gerado grande interesse recentemente, em razão de seu sucesso no mercado, seu alto valor e do grande potencial que representa para a aquicultura (MAZÓN et al., 2007). Ele tem surgido nos últimos anos como uma das espécies promissoras para a aquicultura por uma série de vantagens, tais como: 1) alta taxa de conversão alimentar, incorporando de $40 \%$ a $60 \%$ do alimento ingerido (MANGOLD; BOLETZKY, 1973; WELLS, 1978; MANGOLD, 1983); 2) rápido crescimento (cerca de 3\% de taxa de crescimento diário) (MANGOLD; BOLETZKY, 1973); 3) conteúdo proteico alto, representando $70 \%$ a 90\% do peso seco da composição do seu corpo (O'DOR; WELLS, 1987; LEE, 1994); e 4) alta fecundidade, produzindo de 100 a 500 mil ovos por fêmea (WELLS, 1978; MANGOLD, 1983; IGLESIAS et al., 1997). Os machos alcançam maior tamanho (MANGOLD, 1983; FORSYTHE; VAN HEUKELEM, 1987).

$\mathrm{Na}$ Espanha, a engorda do polvo está apenas no estágio inicial, concentrada ao longo da costa atlântica e no Mediterrâneo (MAZÓN et al., 2007). Os experimentos realizados com alimentos na Galícia (NW Espanha) (IGLESIAS et al., 1997; LUACES; REY, 1999; RODRIGUEZ et al., 2006; CHAPELA et al., 2006) e no Mediterrâneo (AGUADO GIMÉNEZ; GARCÍA GARCÍA, 2002; GARCÍA GARCÍA; CEREZO VALVERDE, 2004, 2006) têm provido resultados excelentes usando uma dieta com misturas de caranguejo, mexilhão e peixe. No entanto, o cultivo desde a fase de ovo até estar subadulto tem sido realizado com sucesso somente em laboratório e em escala experimental (ITAMI et al., 1963; VILLANUEVA, 1995; CARRASCO et al., 2003; IGLESIAS et al., 2004), significando que o cultivo de polvo é atualmente restrito à engorda de subadultos capturados na pesca (MAZÓN et al., 2007).

Considerando-se a importância econômica do polvo comum para o Brasil e o acentuado declínio na sua captura, foi realizada uma revisão sobre informações disponíveis sobre o seu cultivo e especulou-se sobre as possíveis adaptações e inovações que podem ser úteis ao desenvolvimento final de unidades de produção comercialmente rentáveis. Procurou-se somar informações e contribuir para o desenvolvimento da metodologia de seu cultivo comercial.

\section{Produção}

Poucas espécies de cefalópodes são comercialmente capturadas em grande escala (KREUZER, 1984). A lula é a principal espécie de cefalópode, representando $73 \%$ dos cefalópodes capturados no mundo; a sépia (tipo de molusco) é o segundo, com 15\%; e o polvo, o terceiro, com 8,8\% respectivamente. O total de desembarque de cefalópodes chegou ao pico de 3,6 milhões de toneladas em 2000 (VAZ-PIRES et al., 2004).

Nas capturas mundiais de cefalópodes em 2005, destacaram-se China, Japão, Marrocos, Espanha e outros países (MADRID, 2007) (Figura1).

Os principais países consumidores de cefalópodes são Japão, Coreia, Argentina, Taiwan e China, seguidos pelo grupo que inclui Espanha, Portugal, Marrocos, Mauritânia, Grécia e Itália (BALDRATI, 1989; VAZ-PIRES et al., 2004).

Segundo Madrid (2008), os quatro países da Tabela 1 participam com 85\% das importações mundiais de polvo. De acordo com o mesmo autor, deve-se ressaltar que apenas aproximadamente 10\% da produção mundial de polvo está identificada nos dados estatísticos da FAO (2008), como o polvo comum O. vulgaris (37.377 t), ou seja, grande parte da produção pesqueira de polvo constitui-se de espécies de menor valor de mercado e de tamanhos inferiores.

Segundo Madrid (2008), analisando as informações estatísticas da FAO, verifica-se que a produção mundial de polvo proveniente da aquicultura se limita a uma pequena produção da Espanha, equivalente a 11 
toneladas, em 2006, que foi menor que a de 1998, quando foram produzidas 32 toneladas. Segundo o mesmo autor, o Japão, que em 1967 produziu 117 toneladas de polvo cultivado, foi diminuindo paulatinamente até 41 toneladas em 1971, último ano em que figurou nas estatísticas.

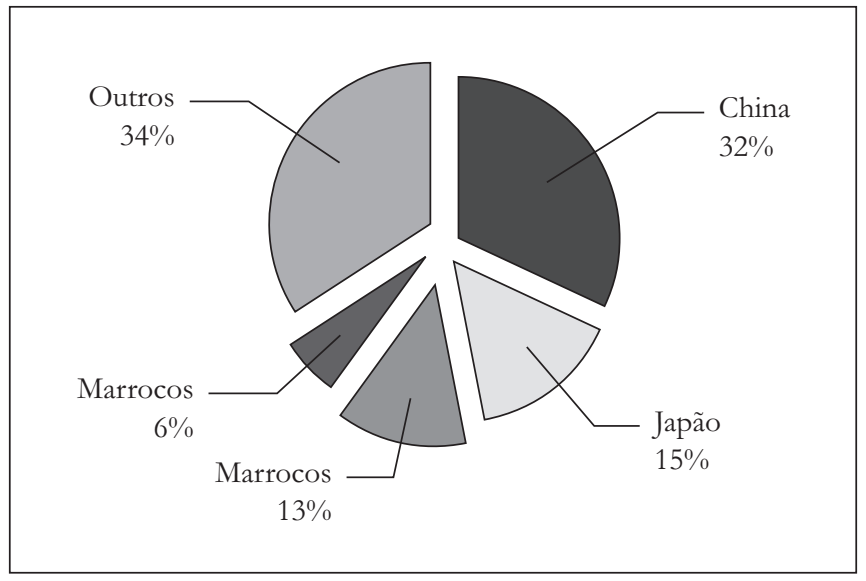

Figura 1 - Captura mundial de polvo, segundo os principais países, em 2005 (359.053 toneladas)

Fonte: MADRID, 2007.

Tabela 1 - Importações mundiais de polvo (em toneladas)

\begin{tabular}{lcc}
\hline País & 2001 & 2006 \\
\hline Japão & 87.050 & 48.400 \\
Itália & 45.800 & 51.200 \\
Coreia & 36.400 & 51.900 \\
Espanha & - & 36.800 \\
\hline
\end{tabular}

Fonte: MADRID, 2008.

No Brasil, segundo Madrid (2007), entre janeiro e novembro de 2007, o valor das exportações de polvo congelado atingiu a quantia de US $\$ 3.270 .000,00$ (775 t), em que Santa Catarina participou com 83\%; seguida por São Paulo (13,3\%); e Rio Grande do Norte, Rio de Janeiro e Rio Grande do Sul, perfazendo juntos 2,87\%. De acordo com o mesmo autor, o preço unitário das exportações catarinenses (US\$ 4,76/kg) foi superior ao das paulistas (US\$2,60/kg). A Espanha destaca-se como o principal comprador do polvo congelado produzido pelo Brasil, com 81,43\% do valor, seguida da Argentina (9,70\%) e de Portugal (5,72\%). Uruguai, Itália, Japão e França participam com os outros 5,72\%.

A Tabela 2 demonstra a evolução das exportações de polvo no Brasil.

Segundo a FAO (2008), da captura mundial de polvo, 10,4\% refere-se ao polvo comum (O. vulgaris), mas $87,2 \%$ são de espécies não identificadas. A captura mundial de polvo O. vulgaris diminuiu de 80.247 toneladas em 1991 para 37.455 em 2005 (FAO, 2008) (Figura 2). No Japão, anualmente são introduzidos de 12.000 a 17.000 potes para fornecer abrigo e melhorar a conservação dos ovos como medida para proteger o estoque pesqueiro da espécie. 
IGARASHI, M. A.

Tabela 2 - Exportações de polvo no Brasil

\begin{tabular}{lcc}
\hline Ano & Volume (t) & Valor (US\$ mil) \\
\hline 2001 & 265 & 605 \\
2002 & 117 & 349 \\
2003 & 296 & 1.037 \\
2004 & 1.241 & 5.324 \\
2005 & 662 & 3.814 \\
2006 & 787 & 2.814 \\
\hline
\end{tabular}

Fonte: FAO, 2007.

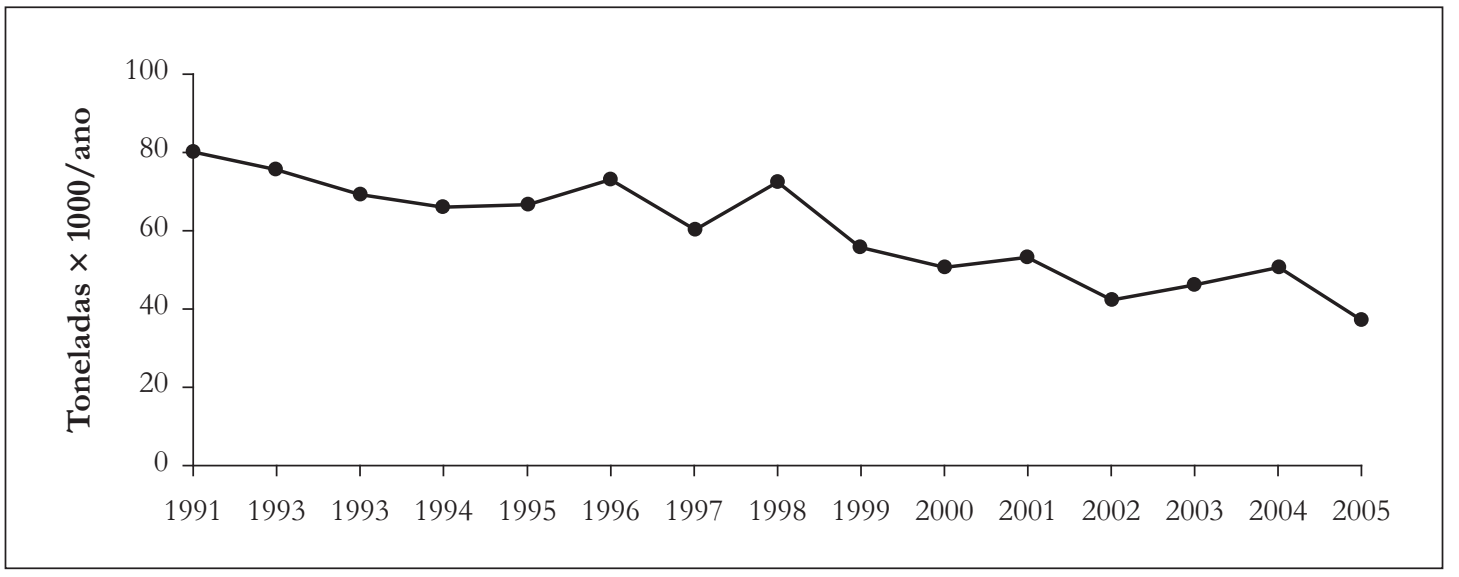

Figura 2 - Captura mundial de polvo Octopus vulgaris

Fonte: FAO, 2008.

\section{Distribuição geográfica}

O polvo é uma espécie com vasta distribuição mundial, por isso é considerado um animal cosmopolita. Ele possui hábitos bentônicos e pode ser encontrado em águas tropicais, subtropicais e temperadas (MATTEAZZI, 2006), sendo abundante no Mar Mediterrâneo, no leste do Oceano Atlântico e em águas japonesas (GUERRA, 1981). Apresenta-se como espécie bentônica e nerítica, ocorrendo da linha costeira à borda externa da plataforma continental, em profundidade de 0 a $200 \mathrm{~m}$, onde é encontrada uma variedade de habitats, tal como rochas, recifes de coral e plantas (VAZ-PIRES et al., 2004). O mimetismo apresentado por essa espécie torna-o um verdadeiro mestre do disfarce (SALGADO, 2000).

\section{Ciclo de vida}

O ciclo de vida do O. vulgaris compreende várias fases sequenciais, desde o embrião, passando pela fase larval, juvenil, subadulto, adulto até o estádio senil (SALGADO, 2000), e tem sido amplamente estudado (NEAF, 1923, 1928; ITAMI et al., 1963; MANGOLD-WIRZ, 1963; NIXON, 1969, 1971; GUERRA, 1979 , 1981; HATANAKA, 1979). Trata-se de um octópode com pequenos ovos (2-3 mm) (MANGOLD, 1983). 
O indivíduo recém-eclodido é planctônico, com peso aproximado de 1,2 mg e comprimento do manto de 1,7 mm (MANGOLD, 1997).

A duração do estágio planctônico de $O$. vulgaris depende da temperatura e varia de 33 a 40 dias quando cultivado na temperatura média de $24,7^{\circ} \mathrm{C}$ (ITAMI et al. 1963) e de 50 a 60 dias a $21^{\circ} \mathrm{C}$ (VILLANUEVA, 1995). Após 12 a 24 meses de crescimento, os adultos pesam de 2 a $10 \mathrm{~kg}$ (MANGOLD, 1997), com machos maturando com 190 g e fêmeas com 1.000 a 2.000 g, no Mediterrâneo (MANGOLD; BOLETZKY, 1973; MANGOLD, 1983).

O ciclo de vida curto, de 12 a 18 meses, o crescimento rápido até 13\% da biomassa/dia e a taxa de conversão alimentar de 15\% a 43\% são consideradas as características básicas mais relevantes que influenciaram o cultivo de O. vulgaris (MANGOLD; BOLETZKY, 1973; MANGOLD, 1983; NAVARRO; VILLANUEVA, 2003). Tal como a maior parte dos cefalópodes é uma espécie carnívora durante todo o seu ciclo de vida (SALGADO, 2000).

O ciclo de vida do O. vulgaris, sob condições de cultivo, foi completado pela primeira vez no ano de 2001 por Iglesias et al. (2002), usando Artemia e zoeas de caranguejo spider crab (Maja squinado) como alimento, em que a sobrevivência durante o cultivo de paralarvas foi de 31,5\% / dia após a eclosão, pesos de 0,5 a 0,6 $\mathrm{kg}$ na idade de seis meses e peso de 1,6 kg dois meses mais tarde (IGLESIAS et al., 2002 apud VAZ-PIRES et al., 2004).

\section{Reprodução}

Os reprodutores de $O$. vulgaris são mantidos na proporção de um macho para uma fêmea; em tanques retangulares com capacidade entre 5 a 10/ $\mathrm{m}^{3}$ (IGLESIAS et al., 2000a); temperatura da água e condições de salinidade estabelecidas em uma faixa de 13 a $20{ }^{\circ} \mathrm{C}$ e 32 a $35 \%$, respectivamente (VAZ-PIRES, et al., 2004); e baixa luminosidade nos tanques para obtenção de desova mais rápida (IGLESIAS et al., 2000a).

O acasalamento ocorre quando o macho libera o esperma (MANGOLD-WIRZ,1963) para o interior da cavidade do corpo da fêmea. Na cópula, os machos inserem o hectocotylus na cavidade interna do manto das fêmeas (VAZ-PIRES et al., 2004). O período da postura é longo, podendo estender-se por todo o ano (WODINSKY, 1972) e a fêmea protege e limpa os ovos até sua eclosão.

A fêmea de O. vulgaris, quando desova, produz um número estimado de 100.000 a 500.000 ovos uma única vez (MANGOLD, 1983). Iglesias et al. (1997) obtiveram um número máximo de 605.000 ovos em experimento de reprodução com polvo.

A morte ocorre após o período reprodutivo para ambos os sexos (MATTEAZZI, 2006). Os machos morrem após a cópula e as fêmeas param de se alimentar durante a incubação e morrem logo após a eclosão dos ovos (RUPPERT; BARNES, 2005 apud MATTEAZZI, 2006).

A estação de desova depende da região em que os animais se encontram e dois picos de desova por ano podem ser observados por meio da distribuição em sua abrangência: no Mediterrâneo, ocorre entre abril e maio, correspondendo ao grupo migrante na costa na primavera (mais importante no Mediterrâneo) e, no Japão, em outubro, correspondendo ao grupo migrante no outono (mais importante no Japão) (FAO, 2003).

A temperatura é descrita como um dos fatores primários no desenvolvimento embrionário em cefalópodes (BOLETZKY, 1989). No Mediterrâneo, Villanueva (1995) observou um período de 34 dias após o início da desova até a primeira paralarva eclodida de $O$. vulgaris, quando a temperatura da água foi elevada para $20 \pm 1{ }^{\circ} \mathrm{C}$. Iglesias et al. (2000a), em condições de laboratório, observaram um período de incubação de 47 dias de 17 a $19^{\circ} \mathrm{C}$ (IGLESIAS et al., 2000a apud VAZ-PIRES et al., 2004).

\section{Cultivo de paralarvas}

Os experimentos de cultivo com paralarva de polvo comum vêm sendo realizados principalmente no Japão (ITAMI et al., 1963; IMAMURA, 1990; HAMASAKI et al., 1991) e na Espanha (VILLANUEVA, 1994, 1995; IGLESIAS et al., 2004) para obtenção de subadultos utilizados no cultivo.

Rev. Acad., Ciênc. Agrár. Ambient., Curitiba, v. 8, n. 4, p. 417-427, out./dez. 2010 
As paralarvas - termo definido por Young e Harman (1988) - de O. vulgaris eclodem com 1,5 a 2,9 $\mathrm{mm}$ de comprimento total (IGLESIAS et al., 2007). Elas são planctônicas, nadam ativamente e têm alta taxa metabólica (VILLANUEVA, 1995; PARRA et al., 2000; IGLESIAS et al., 2000; NAVARRO; VILLANUEVA, 2003). Podem se alimentar de copepodas, crustáceos zoeae e mysidacea, zooplâncton cultivado, Artemia salina, ictioplâncton e, finalmente, de crustáceos zoeae obtidos em laboratório da reprodução de estoques de caranguejo (Carcinus maenas), caranguejo swimming crab (Necora puber) e camarão (Palaemon serratus) (IGLESIAS et al., 2000). Villanueva et al. (2002 apud MAT'TEAZZI, 2006) concluíram que os indivíduos alimentados com náuplios de Artemia a $10 \mathrm{ml}^{-1} / \mathrm{dia}^{-1}$ e microcápsulas apresentaram maior crescimento e atividade proteolítica que os indivíduos alimentados com concentrações menores de Artemia.

Segundo Iglesias et al. (2000a), as paralarvas de O. vulgaris podem ser introduzidas nos tanques de cultivo no mesmo dia da eclosão, sendo cultivadas em uma densidade de 10 paralarvas/litro, com 24 horas diárias de iluminação. De acordo com os mesmos autores, com quatro dias de intervalo o fundo do tanque pode ser limpo e $1 / 4$ do volume de água renovado e a temperatura pode variar de 18 a $20{ }^{\circ} \mathrm{C}$. Microalgas (Isochrysis galbana e Tetraselmis suecica) podem ser introduzidas nos tanques de cultivo, ajustando a concentração em 150.000 células/ml.

No Japão, Itami et al. (1963) foram os pioneiros no cultivo de O. vulgaris e tiveram êxito usando Palaemon serrifer zoeae como sua principal presa. Eles obtiveram juvenis bentônicos aos 33 dias mantendo uma temperatura média de $24,7{ }^{\circ} \mathrm{C}$ e alcançando uma taxa de sobrevivência de $5 \%$ em 60 dias (IGLESIAS et al., 2007).

A duração do período planctônico de $O$. vulgaris depende da temperatura e leva cerca de 47 a 54 dias a $21,2{ }^{\circ} \mathrm{C}$, e 30 a 35 dias a $23{ }^{\circ} \mathrm{C}$ (IMAMURA, 1990; VILLANUEVA, 1995), após o qual os octopuses iniciam o assentamento no fundo (IGLESIAS et al., 2007). Mais especificamente, a fixação das paralarvas no fundo pode acontecer aos 30 ou 40 dias após a eclosão dos ovos, a uma temperatura media da água de $24,7{ }^{\circ} \mathrm{C}$, pesando os animais entre 0,1 e 0,25 g (ITAMI et al., 1963).

Embora o cultivo de paralarvas em escala de laboratório seja possível usando como alimento crustáceos obtidos na natureza (ITAMI et al., 1963), ou zoea de caranguejo cultivada (VILLANUEVA, 1995), as sobrevivências no estágio bentônico de $O$. vulgaris foram relativamente baixas $(8,0 \%$ e $8,9 \%$, respectivamente) (OKUMURA et al., 2005).

\section{Mortalidade}

A grande dificuldade no cultivo de polvo comum é a alta mortalidade que ocorre em sua primeira fase de vida pós-embrionária. Esses indivíduos apresentam atividade metabólica alta e um comportamento alimentar extremamente voraz, podendo entrar em inanição rapidamente na ausência de alimento (MATTEAZZI, 2006).

A alta taxa de mortalidade pode ser atribuída a três fatores: indisponibilidade de uma dieta viva, adequada tanto na composição nutricional como em tamanho; escassez de técnicas apropriadas para o cultivo; escassez de conhecimento da biologia da espécie nessa fase do ciclo de vida (em sua primeira fase de vida pós-embrionária) e, consequentemente, a falta de informação sobre suas necessidades nutricionais básicas (MOXICA et al., 2002).

\section{Engorda}

O crescimento do polvo é influenciado por vários fatores, tais como: temperatura da água, disponibilidade de alimento, tamanho e espécies (FORSYTHE, 1984; MANGOLD; BOLETZKY, 1973; BORER, 1971).

A engorda pode ser realizada com a temperatura da água variando de 13 a 19, $5^{\circ} \mathrm{C}$ (IGLESIAS et al., 2000) e, assim sendo, com todos os dados obtidos, o desempenho ótimo para engorda de O. vulgaris está entre 16 e $21^{\circ} \mathrm{C}$ (VAZ-PIRES et al., 2004). Os estudos realizados no México com O. maya têm demonstrado que, da mesma forma que o O. vulgaris, ele possui atributos próprios, oferecendo à América tropical outra espécie com um grande potencial de cultivo (ROSAS, 2008). 
A salinidade necessária varia entre $32 \%$ a $35 \%$, com fluxo de água de $1,2 \mathrm{~m}^{3} / \mathrm{h}$ (IGLESIAS et al., 2000). O O. vulgaris é uma espécie estritamente marinha, apresentando baixa tolerância a baixas concentrações de sais. Vive na natureza em concentrações de sais de aproximadamente 35\%, sendo a concentração mínima de sal de aproximadamente 27\% (BOLETZKY; HANLON, 1983). Isso significa que flutuações leves na salinidade, em razão da introdução de água doce (por exemplo, proximidade de rios, forte chuva ou água doce das camadas subterrâneas naturais) no sistema de cultivo, podem ser fatais para eles.

O polvo O. vulgaris muda o seu hábito alimentar passando de pequenos crustáceos para crustáceos maiores durante o seu crescimento (MANGOLD, 1983) e rejeita qualquer excesso de alimento (VAZ-PIRES et al., 2004). Embora prefiram alimentos vivos, podem ser adaptados a aceitar alimentos mortos como pedaços de caranguejo, peixes ou moluscos (BOUCAUD-CAMOU; BOUCHER-RODONI, 1983). Os peixes, siris e mexilhões podem ser mantidos congelados e, na véspera, são descongelados e ofertados inteiros aos polvos, dentro de um comedouro fechado, onde nada escapa para o meio ambiente (CARVALHO FILHO, 2004). Em estudos realizados por Aguado Giménez e García García (2002), o consumo de alimento foi maior com dieta à base de caranguejo, mas o peixe foi também eficiente na alimentação.

Vários tipos de alimentos foram testados, incluindo caranguejo (C. maenas, Polybius henslowr) (IGLESIAS et al., 1997, 2000a), sardinhas (Sardina pilchardus) e peixe "boga do mar" (Boops boops) (GARCIA GARCÍA; AGUADO GIMÉNEZ, 2002), dos quais os caranguejos, especialmente quando vivos, parecem ser os mais desejáveis em termos de crescimento (CAGNETTA; SUBLIMI, 2000).

O alimento pode ser suprido uma vez ao dia, compreendendo $80 \%$ de crustáceos $(P$. henslowi, Macropipus corrugatus, C. maenas), 15\% de peixes (Micromesistius poutassou) e 5\% de moluscos congelados (Mytilus sp.) (IGLESIAS et al., 2000). A alimentação é fornecida diariamente, tomando como base $10 \%$ do peso total dos animais (CARVALHO FILHO, 2004). Não há problemas sérios de canibalismo ou competição por alimentos. O crescimento no cultivo em jaulas oscila entre 0,3 a $0,8 \mathrm{~kg} / \mathrm{mês}$ e ocorre baixa mortalidade $(5,7 \%)$ usando alimentos congelados de baixo valor, incluindo sardinha (S. pilchardus), peixe "scad" (Trachurus trachurus), bacalhau blue whiting (M. poutassou), peixe "boga do mar" (B. boops), cavala (Scomber scombrus) e mexilhão (Mytilus sp.) (RAMA-VILLAR et al., 1997).

$\mathrm{Na}$ engorda, o tamanho inicial dos polvos O. vulgaris deve ser similar, e a densidade inicial não deve exceder $10 \mathrm{~kg} / \mathrm{m}^{3}$ (OTERO et al., 1999). Eles também preferem esconderijos sem iluminação interior (ANDERSON et al., 1999). Itami et al. (1963) concluíram que, após os três meses da eclosão, os polvos alcançaram pesos compreendidos entre 11 e $69 \mathrm{~g}$, com uma média de $39 \mathrm{~g}$, podendo atingir o tamanho comercial de 2,5 a $3 \mathrm{~kg}$ (iniciando com indivíduos com $750 \mathrm{~g}$ ) em três ou quatro meses, com mortalidade que não excede $10 \%$ a $15 \%$ (IGLESIAS et al., 2000a).

O processo de engorda leva quatro meses e três ciclos de engorda por ano. O peso de abate recomendado em cultivo com sexos separados é de $3 \mathrm{~kg}$ para machos e 2,5 $\mathrm{kg}$ para fêmeas de O. vulgaris (SANCHES et al., 1998).

Os cálculos em geral indicam que uma companhia com 25 gaiolas seria capaz de produzir cerca de 11.000 polvos de O. vulgaris/ano (IGLESIAS et al., 2000b). A separação dos sexos durante a fase de engorda é recomendada. As fêmeas não fecundadas continuam o crescimento até o tamanho comercial e, em cultivo com sexos separados, os machos crescem mais rápido que as fêmeas (VAZ-PIRES et al., 2004).

\section{Considerações finais}

Os resultados científicos obtidos com o cultivo de polvo indicam que o ciclo de vida do O. vulgaris é compreendido atualmente, mas o cultivo de paralarvas é possível somente em condições de laboratório e a mortalidade é ainda alta. As principais questões para o futuro são as pesquisas nutricionais para as paralarvas e a combinação correta de variáveis físico-químicas como temperatura, salinidade e outros fatores da qualidade da água.

A engorda de indivíduos selvagens subadultos é a fase industrializada do ciclo de vida, em tanques e gaiolas flutuantes. A fácil adaptação em cativeiro e a alimentação baseada em alimentos de baixo valor, assim 
como o rápido crescimento e alto valor comercial do $O$. vulgaris, são as principais razões para ser otimista em relação ao futuro dessa espécie para a aquicultura.

Assim, as pesquisas futuramente devem focalizar métodos de cultivo de paralarvas de O. vulgaris, especialmente sobre alimentos vivos e dietas inertes. Os experimentos sobre a sobrevivência e os estudos direcionados ao desenvolvimento de dietas secas para o crescimento de subadultos também serão de grande importância.

Com a implantação de novas e aperfeiçoadas técnicas poderá ser melhorada a qualidade de vida daqueles que vivem no Brasil, por meio da produção de polvos, na criação de empregos nas referidas regiões em que a produção dessa espécie pode ajudar a atender às necessidades dos mercados de consumo interno e de exportação. Tal medida pode propiciar uma balança de pagamentos e intercâmbio mais favorável para a economia da região.

Portanto, outro objetivo de grande importância seria a reprodução artificial de polvos em laboratório, permitindo criar milhões de jovens e depois repovoar áreas onde a pesca tenha reduzido a níveis próximos da extinção.

A possibilidade de ampliação das atividades ligadas à captura de polvo é finita, em razão das limitações bioecológicas naturais existentes. Diante dessa inevitabilidade, as esperanças se voltam para o povoamento, o repovoamento e o cultivo do polvo.

\section{Referências}

AGUADO GIMÉNEZ, F; GARCÍA GARCÍA, B. Growth and food intake models in Octopus vulgaris Cuvier (1797): influence of body weight, temperature, sex and diet. Aquaculture International, v. 10, n. 5, p. 361-377, 2002.

ANDERSON, R. C. et al. Determination of the diet of Octopus rubescens Berry, 1953 (Cephalopoda: Octopodidae), through examination of its beer bottle dens in puget sound. Malacologia, v. 41, n. 2, p. 455-460, 1999.

BALDRATI, G. Handling, marketing and processing of cephalopods in Italy. Industria Conserve, v. 64, n. 3, p. 353355, 1989.

BOLETZKY, S. V. Recent studies on spawning, embryonic development and hatching in the Cephalopoda. Advances in Marine Biology, v. 25, n. 1, p. 85-115, 1989.

BORER, K. T. Control of food intake in Octopus briareas Robson. Journal of Comparative Physiological Psychology, v. 75, n. 2, p. 171-185, 1971.

BOYLE, P. R.; BOLETZKY, S. V. Cephalopod populations: definition and dynamics. Philosophical Transactions of the Royal Society of London. Series B, v. 35, n. 1, p. 985-1002.

BOUCAUD-CAMOU, E.; BOUCHER-RODONI, R. Feeding and digestion in cephalopods. In: WILBUR, A. S. M. (Ed.). The mollusca. Physiology. London: Academic Press, 1983. part 2, v. 5, p. 149-187.

CAGNETTA, P.; SUBLIMI, A. Productive performance of the common octopus (Octopus vulgaris C.) when fed on a monodiet. Recent advances in mediterranean aquaculture finfish species diversification. Cahiers Options Méditerranéennes, v. 47, p. 331-336, 2000.

CARRASCO, J. F. et al. Cultivo intensivo de paralarvas de pulpo (Octopus vulgaris, Cuvier 1797) utilizando como base de la alimentación zoeas vivas de crustáceos. In: CONGRESO NACIONAL DE ACUICULTURA, 9., 2003, Cádiz. Anais... Cádiz: CNA, 2003. p. 255-256.

CARVALHO FILHO, J. Cultivo de polvos: Epagri inicia cultivo experimental em Santa Catarina. 2004. Disponível em: <http://www.pesca.sp.gov.br/destaque.php? id_destaque=17>. Acesso em: 19 ago. 2007.

CHAPELA, A. et al. Growth of common octopus (Octopus vulgaris) in cages suspended from rafts. Scientia Marina, v. 70, n. 1, p. 121-129, 2006.

Rev. Acad., Ciênc. Agrár. Ambient., Curitiba, v. 8, n. 4, p. 417-427, out./dez. 2010 
FOOD AND AGRICULTURE ORGANIZATION OF THE UNITED NATIONS - FAO. Species 2003, identification sheets. 2003. Disponível em: <http://www.fao.org/figis/servlet/FiRefServlet?ds=species\&fid=3571>. Acesso em: 4 jan. 2008.

FOOD AND AGRICULTURE ORGANIZATION OF THE UNITED NATIONS - FAO. Fao Fishstat 2007, fisheries and aquaculture information and statistics service (FIES). 2007. Disponível em: <http://www.fao.org/ fishery/topic/16073>. Acesso em: 4 jan. 2008.

FOOD AND AGRICULTURE ORGANIZATION OF THE UNITED NATIONS - FAO. Food and Agriculture Organization of The United Nations. Species fact sheets: Octopus vulgaris (Lamarck, 1798). 2008. Disponível em: <http://www.fao.org/website/FIRetrieveAction.do?do>. Acesso em: 17 jan. 2008.

FORSYTHE, J. W. Octopus joubini (Mollusca: Cephalopoda): a detailed study of growth through the full life cycle in a closed seawater system. Journal of Zoology, v. 202, n. 3, p. 393-417, 1984.

FORSYTHE, J. W.; VAN HEUKELEM, W. F. Growth. In: BOYLE, P. R. (Ed.). Cephalopod life cycles. London: Academic Press, 1987. v. 2, p. 351-365.

GARCÍA GARCÍA, B.; CEREZO VALVERDE, J. Influencia del número de días de ayuno a la semana sobre el crecimiento, el índice de conversión y la superviviencia en el pulpo de roca (Octopus vulgaris Cuvier, 1797). Revista AquaTIC, n. 21, 2004.

GARCÍA GARCÍA, B.; AGUADO GIMÉNEZ, F. Influence of diet on ongrowing and nutrient utilization in the common octopus (Octopus vulgaris). Aquaculture, v. 211, n. 1/4, p. 171-182, 2002.

GUERRA, A. Aspectos da biología do polbo comum. Boletin de la Sociedad de la Galicia de Historia Natural, v. 3, n. 2, p. 75-79, 1979.

GUERRA, A. Spatial distribution pattern of Octopus vulgaris. Journal Zoological of London, v. 195, n. 1, p. 133-146, 1981.

HAMASAKI, K. et al. Effect of marine microalgae Nannochloropsis sp. on survival and growth on rearing pelagic paralarvae of Octopus vulgaris, and results of mass culture in the tank of 20 metric tons. Saibai-Giken, v. 19, n. 1, p. 75-84, 1991.

HATANAKA, H. Studies on the fisheries biology of common octopus of the northwest coast of Africa. Bulletin Farming Seas Fisheries Research Lab, v. 17, n. 1, p. 13-124, 1979.

IGLESIAS, J. et al. Primeras experiencias sobre el cultivo integral del pulpo (Octopus vulgaris, Cuvier) en el Instituto Español de Oceanografía. In: CONGRESO NACIONAL DE ACUICULTURA, 6., 1997, Cartagena, Spain. Anais... Cartagena, Spain: CAN, 1997. p. 221-226.

IGLESIAS, J. et al. Culture of octopus (Octopus vulgaris, Cuvier): present knowledge, problems and perspectives. 2000. p. 313-321. Disponível em: <http:// ressources.ciheam.org/om/pdf/c47/00600632.pdf>. Acesso em:19 ago. 2007.

IGLESIAS, J. et al. Culture of octopus (Octopus vulgaris, Cuvier): present knowledge, problems and perspectives. Recent advances in mediterranean aquaculture finfish species diversification. Cahiers Options Méditerranéennes, v. 47, p. 313-322, 2000a.

IGLESIAS, J. et al. Ongrowing, reproduction and larvae rearing of octopus (Octopus vulgaris Cuvier), a new candidate for aquaculture in Galicia (NW Spain). In: WORKSHOP ON NEW SPECIES FOR AQUACULTURE, 1., Faro. Anais... Faro: WSA, 2000b. p. 20-21.

IGLESIAS, J. et al. The completed life cycle of the Octopus (Octopus vulgaris, Cuvier) under culture conditions: paralarval rearing using Artemia and zoeae, and first data on juvenile growth up to 8 months of age. Aquaculture International, v. 12 , n. $4 / 5$, p. $481-487,2004$.

IGLESIAS, J. et al. Rearing of Octopus vulgaris paralarvae: present status, bottlenecks and trends. Aquaculture, v. 266, n. 1/4, p. 1-15, 2007. Disponível em: <http//www. elsevier.com/locate/aqua-online>. Acesso em: 17 jan. 2008. 
IGLESIAS, J. et al. Paralarvae culture of octopus (Octopus vulgaris) using artemia and zoeas, and first data on juvenile growth up to eight months of age. Seafarming today and tomorrow-extended abstracts and short communications. European Aquaculture Society - EAS, Oostende, p. 268-269, 2002.

IMAMURA, S. Larval rearing of octopus (Octopus vulgaris Cuvier). The process of technological development and some problems remained. Collect. Breeding, v. 52, n. 1, p. 339-343, 1990.

KREUZER, R. Cephalopods: handling, processing and products. Roma: FAO, 1984.

LEE, P. G. Nutrition of cephalopods: fuelling the system. Marine and Freshwater Behaviour and Physiology, v. 25, n. 1/3, p. 35-51, 1994.

LUACES, M.; REY, M. El engorde industrial de pulpo (Octopus vulgaris) en jaulas: análisis de dos años de cultivo en la Ría de Camariñas (Galicia). In: FERNÁNDEZ-PALACIOS, H.; IZQUIERDO, M. (Ed.). Convergencia entre investigación y empresa: un reto para el Siglo XXI. Las Palmas de Gran Canaria: ICCM, 1999. n. 4, p. 184-268.

MADRID, R. M. Produção mundial de polvo. Polvo News, ano 1, n. 1, p. 2, 2007.

MADRID, R. M. A potencialidade do cultivo de polvo na aqüicultura brasileira. 2008. Disponível em: < http:// www.panoramadaaquicultura.com.br/paginas/revistas/107/polvo107.asp>. Acesso em: 9 jun. 2008.

MANGOLD, K.; BOLETZKY, S. V. New dates on reproductive biology and growth of Octopus vulgaris. Marine Biology, v. 19 , v. 1, p. $7-12,1973$.

MANGOLD, K. Food, feeding and growth in cephalopods. Memoirs of the National Museum of Victoria, v. 44, n. 2, p. 81-93, 1983.

MANGOLD, K. Octopus vulgaris: review of the biology. In: WORKSHOP ON: THE FISHERY AND MARKET POTENTIAL OF OCTOPUS IN CALIFORNIA, 1997, Washington, DC. Proceedings... Washington, DC: Smithsonian Institution, 1997. p. 85-90.

MANGOLD-WIRZ, K. Biologie des céphalopodes benthiques et nectoniques de la Mer Catalane. Vie et Milieu, suppl. 13, p. 1-285, 1963.

MATTTEAZZI, L. Cultivo de polvo comum Octopus Vulgaris (Cuv7). 2006. 18 f. Monografia (Especialização em Biologia Marinha) - Universidade de Taubaté, Taubaté, 2006.

MAZÓN, M. J. et al. Evaluation of environmental nitrogen and phosphorus contributions as a result of intensive ongrowing of common octopus (Octopus vulgaris). Aquaculture, v. 266, n. 1/4, p. 226-235, 2007.

MOXICA, C. et al. Cultivo intensivo de paralarvas de pulpo, de Octopus vulgaris Cuvier, 1797, em tanques de $9 \mathrm{~m}^{3}$. Boletín del Instituto Español de Oceanografia, v. 18, n. 1/4, p. 31-36, 2002.

NAVARRO, J. C.; VILLANUEVA, R. The fatty acid composition of Octopus vulgaris paralarvae reared with live and inert food: deviation from their natural fatty acid profile. Aquaculture, v. 219, n. 1/4, p. 613-631, 2003.

NEAF, A. Die cephalopoden. Fauna Flora Golf Neapal, v. 35A, n. 1, p. 149-863, 1923.

NIXON, M. The lifespan of Octopus vulgaris. Proceedings of the Malacologigal Society, v. 38, n. 1, p. 529-540, 1969.

O'DOR, R. K.; WELLS, M. J. Energy and nutrient flow. In: BOYLE, P. R. (Ed.). Cephalopod life cycles. London: Academic Press, 1987. v. 2, p. 109-133.

OKUMURA, S. et al. Improved survival and growth in Octopus vulgaris paralarvae by feeding large type Artemia and Pacific sandeel, Ammodytes personatus. Aquaculture, v. 244, n. 1/4, p. 144-157, 2005.

OTERO, J. J. et al. Engorde de pulpo (Octopus vulgaris Cuvier) a diferentes densidades de estabulación. Libro de resumenes. In: CONGRESO NACIONAL DE ACUICULTURA, 7., 1999, Las Palmas de Gran Canaria. Anais... Las Palmas de Gran Canaria: CNA, 1999. 
PARRA, G. et al. Respiration rates in late eggs and early hatchlings of the common octopus, Octopus vulgaris. Journal of Marine Biology, v. 80, n. 1, p. 557-558, 2000.

RAMA-VILLAR, A. V. etal. Engorde de pulpo (Octopus vulgaris) en batea. In: ACTAS DEL VI CONGRESO NACIONAL DE ACUICULTURA, 6., 1997, Cartagena. Anais... Cartagena: CNA, 1997.

RODRIGUEZ, C. et al. Common octopus (Octopus vulgaris Cuvier, 1797) juvenile ongrowing in floating cages. Aquaculture, v. 254, n. 1/4, p. 293-300, 2006.

ROSAS, C. Cultivo de polvo: desafios e perspectivas para latinoamérica. Polvo News, ano 1, n. 1, p. 3, 2008.

SALGADO, M. Ficha do polvo comum. Naturlink. 2000. Disponível em: < http:// naturlink.sapo.pt/article.aspx?me nuid $=55 \&$ cid $=8881 \& b l=1 \&$ viewall $=$ true $>$. Acesso em: 19 ago. 2007 .

SÁNCHEZ, F. J. et al. Growth of octopus (Octopus vulgaris) males and females under culture conditions. EAS Special Publication, n. 26, p. 244-245, 1998.

SWEENEY, M. J.; ROPER, C. F. E. Classification, type localities and type repositories of recent cephalopoda. In: VOSS, N. A.; VECCHIONE, M; TOLL, R. B. Systematic and biogeography of cephalopods. Washington, DC: Smithsonian Institution Press, 1998. v. 2. p. 561-582. (Smithsonion Contributions to Zoology).

VAZ-PIRES, P. et al. Aquaculture potential of the common octopus (Octopus vulgaris Cuvier, 1797): a review. Aquaculture, v. 238, n. 1/4, p. 221-238, 2004.

VILLANUEVA, R. Decapod crab zoeae as food for rearing cephalopod paralarvae. Aquaculture, v. 128, n. 1/4, p. 143-152, 1994.

VILLANUEVA, R. Experimental rearing and growth of planktonic Octopus vulgaris from hatching to settlement. Canadian Journal of Fisheries and Aquatic Sciences, v. 52, n. 11, p. 2639-2650, 1995.

VILLANUEVA, R. et al. Growth and proteolitic activity of Octopus vulgaris paralarvae with different food rations during first feeding, using Artemia nauplii and compound diets. Aquaculture, v. 204, n. 1/4, p. 269-286, 2002.

WELLS, M. J. Octopus: physiology and behaviour of an advanced invertebrate. London: Chapman and Hall, 1978.

WODINSKY, J. Breeding season of Octopus vulgaris. Marine Biology, v. 16, n. 1, p. 59-63, 1972.

YOUNG, R. E.; HARMAN, R. F. 'Larva', 'paralarva' and 'subadult' in cephalopod terminology. Malacologia, v. 29, n. 1, p. 201-207, 1988.

Recebido: $15 / 08 / 2008$

Received: 08/15/2008

Aprovado: $20 / 10 / 2010$

Approved: 10/20/2010

Rev. Acad., Ciênc. Agrár. Ambient., Curitiba, v. 8, n. 4, p. 417-427, out./dez. 2010 